\title{
st \\ Los estudios sobre el ambiente y la ciencia ambiental
}

\author{
Amelia Nancy Giannuzzo
}

\begin{abstract}
电
RESUMEN

La existencia de la ciencia ambiental es reconocida en libros, revistas de publicación científica y carreras de grado y posgrado. Sin embargo, se desconoce su existencia en forma literal o indirecta, al no ser considerado su aporte, por ejemplo, en los planteos referidos sobre la ciencia y la tecnología de la sustentabilidad. En este trabajo se presentan estos antecedentes, relacionándolos con el objetivo del mismo, que es el de aportar a la dilucidación de la existencia y conformación de la ciencia ambiental. Para esto, se analiza la relación de las disciplinas con la dimensión compleja del ambiente como objeto de estudio y aspectos metodológicos derivados. A los fines de aportar al esclarecimiento conceptual, se identifican las distintas acepciones de ambiente comúnmente referidas en la bibliografía. Además, se discuten aspectos relacionados de multidisciplinariedad, interdisciplinariedad y transdisciplinariedad, y sobre el status epistémico de la ciencia ambiental. Se concluye que una mayor precisión conceptual embasada en un marco compartido por las disciplinas que estudian el ambiente, incluida la ciencia ambiental, y los distintos actores involucrados en las problemáticas ambientales, favorecerá el refinamiento de las metodologías tendientes a disminuir la fragmentación de las investigaciones concernientes y las aplicaciones para su resolución.
\end{abstract}

PALABRAS-CLAVE $\bullet$ Estudios ambientales. Ciencia ambiental. Interdisciplinariedad.

\section{INTRODUGGIóN}

En los últimos años, se ha comenzado a discutir sobre la ciencia y la tecnología de la sustentabilidad, intentando solucionar problemas de orden teórico y práctico, especialmente referidos a la integración de las disciplinas en los estudios sobre el ambiente.

Paralelamente, poco o nada se alude en esos trabajos a la existencia de la ciencia ambiental, y el aporte que puede significar esta área disciplinar, y el de los profesionales formados en la misma, a tales discusiones. Sucede que, por un lado, se la da por reconocida, esto lo demuestra la existencia de libros, revistas de publicaciones científicas y carreras de grado y posgrado en el mundo dedicadas a las ciencias ambientales y, por otro, se desconoce su existencia en forma literal o indirecta, no considerándola en los planteos referidos. 
Por este motivo, el objeto de este trabajo es aportar a la dilucidación de la existencia y conformación de las ciencias ambientales. A continuación, se presentan los planteos y tendencias actuales, para luego relacionarlos con los de este trabajo. Por considerarlo una síntesis de los antecedentes sobre la temática, se ha tomado como base el documento "Desarrollo sustentable: desafios epistemológicos a la ciencia e tecnología" (Modvar \& Gallopín, 2010), el cual se traduce desde una lectura integrada y personal, respetando sus lineamientos generales, pero reordenándolos.

\section{ANTEGedentes: PLANTEOS AGTUALES SOBRE LA GienGiA Y LA TEGNOLOGÍA RELAGIONADOS A LAS INVESTIGAGIONES AMBIENTALES}

Los actuales planteos sobre la ciencia y la tecnología relacionados a las investigaciones ambientales, enmarcados en la problemática sostenible del desarrollo, se basan principalmente en dos facetas: en cambios en la comprensión del mundo, y en cambios en la naturaleza de la toma de decisiones.

Respecto a los cambios en la comprensión del mundo, los mismos están relacionados al conocimiento del comportamiento de los sistemas complejos, incluyendo la idea de incertidumbre en el nivel macroscópico, los que se señalan como cambios en las concepciones epistemológicas ocurridas en parte de los científicos modernos.

La complejidad, también, está asociada al aumento del número de dimensiones utilizadas para definir los problemas y soluciones. Por lo que se han señalado cambios en la naturaleza de la toma de decisiones en muchas partes del mundo, relacionados a un estilo más participativo incluyendo a nuevos actores sociales, como las organizaciones no gubernamentales, junto a la incorporación de nuevos criterios y valores en relación al ambiente, los derechos humanos, las cuestiones de género y otros.

De este modo, dado que un análisis por separado de los subsistemas ecológicos y los sociales no proporciona una suficiente comprensión del conjunto, la unidad de investigación debe incluir el acoplado socio-ecológico del sistema. Como es sabido, la complejidad, no linealidad y auto-organización caracterizan a estos sistemas.

En los sistemas complejos pueden observarse jerarquías, en el sentido de que cada elemento del sistema funciona como un subsistema del mismo, y el propio sistema como un subsistema de un orden mayor. Dado el acoplamiento que suele presentarse entre los diferentes niveles, el sistema debe ser analizado o gestionado en más de una escala en forma simultánea. El reto consiste en el tratamiento transversal de la dinámica de escala, así como la necesidad de articular acciones en diferentes escalas de lo local a lo global. 
Además del manejo de múltiples escalas, la conciliación de las variables cualitativas y cuantitativas, que caracteriza a esos sistemas, se presenta como una dificultad a ser superada.

Según lo antedicho, la ciencia y la tecnología para el desarrollo sostenible deben ser interdisciplinarias por necesidad. La integración de la investigación científica en términos de pertinencia para la toma de decisiones requiere un enfoque holístico y un estilo de investigación interdisciplinaria dada la naturaleza de los sistemas socio-ecológicos como unidad de análisis, que se contrapone al carácter compartimentado de las disciplinas como unidades de comprensión. La identificación y comprensión de causas y de vínculos entre las causas de las problemáticas, asi como la comprensión de la dinámica del sistema es esencial en este contexto. En este sentido se ha asociado la complejidad a la transdisciplinariedad en relación a:

(a) la investigación, desde una perspectiva participativa, como forma de investigación-acción;

(b) las realidades investigadas, caracterizadas por fenómenos irreducibles a una sola dimensión, dependientes del contexto;

(c) el esfuerzo intelectual para comprenderlas, a través de la elaboración de modelos que tomen en cuenta el contexto, así como las relaciones entre sus elementos constituyentes.

Respecto a la incertidumbre asociada al estudio de los sistemas complejos, desde los planteos de una ciencia para la sostenibilidad, se expresa que la comprensión y el conocimiento de los procesos de auto-organización no son sinónimos de la capacidad de predecir. Las fuentes de incertidumbre son diversas: falta de datos, datos inadecuados, definición imprecisa del sistema y sus fronteras, comprensión limitada del sistema, derivada de procesos no lineales como el comportamiento caótico. Frente a la incertidumbre, deben realizarse nuevas investigaciones y diseños de estrategias que no se limiten a reconocer el riesgo, sino a incluirlo. Dada las características de los sistemas complejos, estos escapan a la capacidad humana de control, y el aumento de los conocimientos puede incluso generar más incertidumbre. La existencia de riesgo tiene que ser asumida como una parte natural de la realidad, y no como una excusa para la falta de acción.

Dadas las condiciones descriptas, además, de planteos metodológicos sobre el manejo de múltiples escalas, la conciliación de variables cualitativas y cuantitativas, cuestiones asociadas a la incertidumbre y el riesgo inherente, y sobre la integración de las disciplinas, interesan otros aspectos como, por ejemplo, la necesidad de reexaminar los criterios de validación de hipótesis y otras normas científicas, y la incorporación de otros conocimientos y perspectivas desde otros actores sociales, según crite- 
rios de verdad y calidad más amplios, pero no menos sólidos y rigurosos. Sobre este último punto, la pregunta que se formula es: ¿En qué grado, en qué situaciones, qué tipo y en qué forma alternativa estos conocimientos deben incorporarse?

También se plantea la necesidad de llevar a cabo experiencias innovadoras sobre la manera de generar diálogo entre la ciencia y la política, reconociéndose que la producción científica, en muchos casos, no responde al tipo de conocimientos que puedan ser utilizados por los encargados de formular políticas (Baskerville apud Modvar \& Gallopín, 2010). Esto sucede porque, en general, las cuestiones científicas se plantean de manera estrecha, según escalas de trabajo incompatibles con las que se requieren para la toma de decisiones.

\section{El Ambiente, y SU Dimensión COMPLEJA, COMO OBJETo DE ESTUdio}

El ambiente definido - por ejemplo, en la I Conferencia de las Naciones Unidas sobre el Medioambiente Humano, realizada en Estocolmo, en 1972, como "el conjunto de elementos físicos, químicos, biológicos y de factores sociales, capaces de causar efectos directos o indirectos, a corto o largo plazo, sobre los seres vivos y las actividades humanas" - es objeto de estudio, de manera más o menos directa, en algún o algunos aspectos, de muchas de las disciplinas del conocimiento, entre las cuales deben citarse: la biología, la ecología, la química, la geología, la antropología, la medicina, la geografía, la meteorología, la sociología, la psicología, la economía, el urbanismo, la filosofía, el derecho, la ingeniería, la política.

Incluso, antes de que el concepto de ambiente, formalmente enunciado, originara replanteos epistemológicos y metodológicos desde las disciplinas, esos aspectos ya eran objeto de estudio desde la perspectiva de cada especialidad. Esto se explica por la condición de complejidad de los sistemas ambientales, definida por sus componentes heterogéneos en interacción, que determina que sus subsistemas pertenezcan a dominios conceptuales de distintas disciplinas. Por otra parte, es sabido que el hecho de que los diversos aspectos de un conjunto sean abordados desde las especializaciones disciplinares pertinentes, mediante su correspondiente bagaje histórico, epistemológico, y metodológico, resulta no sólo conveniente, sino ineludible, para la profundización de su conocimiento. Esto explica la paradoja resultante del paralelismo entre especialización y necesidad de diálogo interdisciplinario, en especial, en la convergencia de los estudios ambientales.

Retomando el concepto de ambiente, el conjunto de elementos físicos, químicos, biológicos y de factores sociales está delimitado por la función; o sea, por la capacidad de causar efectos sobre los seres vivos y las actividades humanas. Esos elemen- 
tos, además de ser heterogéneos entre sí, expresan funciones en relación a la conformación del conjunto. Así, las funciones que expresa cada elemento dentro del conjunto, como las que expresa el conjunto, son dependientes entre sí (cf. García, 1994). El grado de dependencia de tales funciones está establecido por el grado de las relaciones dadas entre los elementos y entre el conjunto de elementos físicos, biológicos etc. con otros conjuntos de órdenes equivalentes en ese caso, los seres vivos y las actividades humanas. Una mayor riqueza de relaciones y, por ende, una mayor dependencia de funciones entre los elementos y del conjunto con otros conjuntos, comprendidas a su vez en diversas escalas espaciales y temporales, supone una mayor complejidad.

Dada las dependencias establecidas, es esperable que una alteración producida en el conjunto de elementos, en los elementos, en las relaciones, o en un fragmento de la estructura del gran sistema ambiental se propague a través de la red de relaciones, provocando a su vez alteraciones que estructuren nuevas organizaciones, distintas respecto de las primeras. La graduación de los cambios producidos puede depender del grado de alteración, del tipo de alteración, del momento de ocurrencia, de propiedades como la resiliencia y la dinámica evolutiva y, como ya se expresó, de la localización de la alteración. Por otra parte, la o las causas de la alteración, pueden ser anticipadas, pueden ignorarse por completo o pueden conocerse sólo algunas. El ambiente presenta en forma continua problemas imprevisibles, pero generalmente explicables a posteriori (cf. Mayer, 1998).

El gran sistema ambiental está configurado por la integración de la atmósfera, la hidrosfera, la litosfera, la biosfera y la tecnosfera. En este sentido, las regiones más prístinas del planeta han sido intervenidas por el hombre, al menos a través de acciones de preservación o conservación. Contrariamente, aun las zonas más inhóspitas de las regiones polares son sumidero de compuestos orgánicos sintéticos como los bifenilos policlorados, conocidos como PCBs. Son numerosos los ejemplos de cambios por adaptación que la actividad humana ha generado en otras especies por modificación de sus habitats y otras causas. El cambio climático global es otro de los innegables ejemplos de la afectación de la naturaleza por las actividades humanas, y de la interdependencia de los subsistemas atmósfera - litosfera - biosfera - tecnosfera.

No obstante la evidencia, dada justamente la dimensión compleja de lo ambiental, y pese a los intentos de contenerla en una concepción universalizada por parte de innumerables trabajos de reflexión e investigación, tendientes a resolver diversos planteos de orden tanto teórico como práctico, se observa que ésta es redefinida desde los distintos ámbitos desde los que es abordada con el consecuente "sesgo" epistemológico. Así, en general, en un intento de síntesis, resulta siendo demarcada en forma tangente a un contexto mayor, desde la perspectiva a partir de la cual es aludida. De este modo, los dominios implícitos como el natural, el social, el filosófico, son 
imbricados en ordenamientos que varían en su estructura y relación, de acuerdo a las distintas posiciones. Esto resulta en una pluralidad de concepciones que, dadas las asimetrías en la ponderación de sus componentes, debido a las distintas naturalezas de los enfoques, no siempre son comparables.

Sin embargo, la complicación mayor no reside en la existencia de esta variedad de concepciones entre las distintas disciplinas, y dentro de las mismas, la que en sí, también, entraña facetas beneficiosas como el enriquecimiento conceptual promovido por la pluralidad (Ransanz, 1997). La complicación mayor reside en el riesgo de desconocer, desde el fundamento de las distintas disciplinas, relaciones que afecten al o a los aspectos o componentes por ellas estudiados, las que, a la vez, puedan incidir en el conocimiento de los mismos. De manera recíproca, la profundización en el conocimiento de un componente puede incidir en el conocimiento de sus interacciones con algunos, o el resto de los componentes, incidiendo a su vez, en la solución de las problemáticas en las que están implicados.

Así, otra vez, la misma condición de complejidad, que comprende el estudio del ambiente desde las distintas disciplinas, reivindica la necesidad, o al menos la conveniencia, de una visión integradora de las partes y sus interacciones. Es decir, un consenso "macro" abarcador, desde el cual sea posible abordar el estudio de los componentes y sus interacciones en relación al "todo".

Es preciso remarcar que, si bien se considera conveniente una redefinición de sus aspectos esenciales, la composición del "todo ambiental" sigue siendo un devenir a ser revelado, reconocido, consensuado, no una construcción última o inamovible, por lo que toda redefinición se esbozará desde una posición abierta a nuevos planteos y configuraciones.

Justamente, se insistirá en que la naturaleza compleja del ambiente trasciende las visiones parciales que intenten contenerla. Trasciende, también, al diálogo entre las visiones parciales e, incluso, de existir, a la visión integradora a la que aspiraría una ciencia ambiental. Precisamente, la intención de esclarecimiento que sostiene este trabajo no desconoce los planteos actuales sobre el saber ambiental (cf. Leff, 2010), sino que, dentro de éste, alude principalmente a su matiz científico en relación a la existencia de una ciencia ambiental. Sin embargo, se procura reconocer otros matices relacionados, ya que la evolución científica entendida como la sucesión histórica de cambios en los fundamentos de las ciencias, como es sabido, y como aquí se comprende, en un sentido amplio, propugnará sus fronteras sobre la no-ciencia, o sobre conocimientos no científicos. Así, ciertas definiciones conllevarán ciertas imprecisiones asociadas, por ejemplo, al eco provocado por la confluencia con otras formas de expresiones humanas como la religión o el arte, a las formas o el fondo de su filosofía implícita, a un estadio histórico, o al contexto en el que se expresa. 
Retomando el planteo anterior, esa "macro" aprehensión debiera poder ser articulada a las diversas "micro" aprehensiones de las distintas partes, asegurando procesos fluidos de comunicación que posibilitarán la mejor comprensión de las partes y del todo, y viceversa; y de las identidades, los procesos y las funciones implícitas.

La complejidad del ambiente, además, como se expresó, muestra aspectos cambiantes y de evolución escasamente o nada predecibles, asociados a cambios originados continuamente por la actividad del hombre, y la reacción y adaptabilidad de la naturaleza ante los mismos. Éstos suceden en lapsos, tanto inmediatos como extensos, los que a su vez originan readaptaciones culturales que orientan nuevas posiciones frente a la naturaleza, y reorientan muchas de las actividades humanas.

De este modo, la complejidad del ambiente es a la vez una complejidad cambiante y dinámica, cuyo estudio requiere la articulación de "macro" y "micro" escalas espaciales y temporales, cuya valoración, además, varía en las distintas disciplinas (cf. Reboratti, 2001). Y según veníamos exponiendo se suma, además, la articulación de conocimientos, entendidos como conceptos, percepciones y metodologías de abordaje, de diversas áreas disciplinares, y de la consideración de la pertinencia de conocimientos no científicos en algunas problemáticas.

La necesidad de discernimiento de estas cuestiones fue evidenciada en los últimos treinta y cinco años, particularmente a partir del informe Brundtlan, por una gran diversidad de autores de las distintas disciplinas que comenzaron a intentar dilucidar la ubicación de su objeto de estudio en la esfera de lo ambiental, y a sentar su cimiento epistemológico respecto de la misma. No obstante, en muchos casos, se advierten en tales esfuerzos resonancias de la imprecisión, originadas por la ausencia de un fundamento epistemológico concensuado sobre lo ambiental.

De manera más simple, esto se explica observando cómo, en los debates sobre lo ambiental, se parte de suponer que el concepto de ambiente es universalmente compartido por los referentes o actores implicados. Sin embargo, no siempre es difícil advertir la asimetría conceptual antes aludida, no sólo entre las distintas disciplinas sino dentro de los discursos de los referentes de una misma disciplina. Esto, para quienes interesa el esclarecimiento de esta temática, crea una sensación semejante a la experimentada cuando en una habitación "todos hablan a la vez”. Este último aspecto, en sí mismo, presenta sus propias facetas de interés. Tal vez, esta situación pueda ser explicada por la perturbación que produce el contraste del objeto de estudio respecto de un objeto mayor, puesto de manifiesto desde fuera de los planteos internos de la disciplina de la que se trate, produciendo de algún modo cambios en las concepciones iniciales.

Igualmente, el ejercicio de contrastar el propio objeto de estudio dentro de un objeto mayor puede haber surgido desde el interior de la disciplina, ante la existencia de ciertos límites conceptuales advertidos en las prácticas de investigación y sus apli- 
caciones, como intento de superar algunos planteos acerca de los alcances epistemológicos originariamente definidos dentro de la misma.

Una tercera posibilidad es que ambas situaciones se hayan dado simultáneamente. Esto, a su vez, puede resultar en un mismo momento y/o proposiciones conceptuales coincidentes o, por el contrario, en momentos distintos y/o proposiciones conceptuales contrastantes.

En los tres casos aludidos, la perturbación origina una reacción de acomodamiento al conflicto, que no siempre significa una única resolución del mismo, pudiendo coexistir varias expresiones posibles como respuesta. Por ejemplo, un problema ambiental, como la contaminación de una cuenca hídrica, ofrece a distintas disciplinas variados aspectos de estudio. Sin embargo, tales estudios pronto reconocerán sus alcances y límites conceptuales y metodológicos, si se plantean el objetivo de aportar a la solución integral del problema por separado. De este modo, el "problema ambiental" presenta no sólo un problema para su resolución teórica sino, también, y esencialmente, para su resolución práctica. Es decir, el campo de "lo ambiental" incluye a tales disciplinas, y la validez de los resultados tendrá un carácter relativo, fragmentario o contextual, si no están integrados al resto de los resultados requeridos de las demás disciplinas.

De este modo, las más de las veces, los resultados "parciales" logrados por cada disciplina tendrán un alcance restringido en la aplicación, originando a su vez planteos teóricos que tienden a la apertura y el diálogo con otras disciplinas, y a la revisión de conceptos y fundamentos epistémicos. Sin embargo, está claro que, si bien tales estudios fragmentarios poco aportan a la solución de la problemática, sí, inciden positivamente en avances para el desarrollo interno de las disciplinas que los llevan a cabo, motivo por los que se los siguen realizando.

Respecto a esto último, esta observación resulta uno de los precedentes para la crítica acerca de que el desarrollo de la ciencia no siempre está en concordancia con el desarrollo humano o la conservación de la naturaleza y el ambiente, derivándose diversos planteos éticos. Concretamente, no siempre los estudios sobre el ambiente derivan en mejoras ambientales, observándose una falta de articulación entre las problemáticas ambientales, la investigación y las acciones necesarias a la solución. No casualmente, en los últimos años, se ha ampliado el espectro de espacios para trabajos de reflexión e investigación destinados a planteos relacionados a estas cuestiones. Particularmente, como se aludió en la revisión de antecedentes de este escrito, son dignos de mención los trabajos dedicados a discusiones epistemológicas vinculadas, por ejemplo, a los desafíos planteados por el desarrollo sostenible, entre los que cuenta la relación ciencia-política. 
En síntesis, hasta aquí, se ha puesto en evidencia: (a) el contraste del abordaje histórico del propio objeto de estudio por parte de las distintas disciplinas con la necesidad de "re-conocer" la dimensión compleja de un objeto mayor, que no sólo lo contiene sino que incide a través de múltiples interacciones en el objeto mismo, y en el conocimiento de éste, y de identidades, procesos y funciones asociados; (b) la tensión creada por esta relación recíproca, marcadamente dinámica en sus variados aspectos, que, al potenciar las variadas posibilidades de respuesta de diversas áreas del conocimiento, ha motivado una situación de "todos hablan a la vez", que aún tarda en sosegarse.

Así, dados los diferentes enlaces históricos que relacionan a cada disciplina con el reconocimiento de lo ambiental, que incluyen los flujos de producción, comunicación y divulgación de conocimiento dentro de las mismas, coexisten internamente y en diálogo interdisciplinario las asimetrías conceptuales a las que hasta aquí nos referimos. Esas asimetrías, a su vez, inciden en aspectos epistemológicos y metodológicos implicados en desarrollos teóricos y aplicaciones prácticas relacionadas.

Finalmente, esto se comprende mejor al observar que, en la actualidad, existen especializaciones de las disciplinas como la biología ambiental, la química ambiental, la toxicología ambiental, la ingeniería ambiental, la economía ambiental, la antropología ambiental, la historia ambiental, la sociología ambiental y hasta la ecología ambiental! Y también existen especializaciones como la economía ecológica. Particularmente, la antropología ambiental es denominada indistintamente, también, antropología ecológica. Al respecto, puede observarse que incluso dentro de las mismas especializaciones, los autores difieren en el marco conceptual que incluye lo ecológico dentro de lo ambiental o, por el contrario, lo ambiental dentro de lo ecológico. Frecuentemente, de esta distinción elemental se derivan ambigüedades que orientan las investigaciones y sus aplicaciones, como así también procesos educativos y de divulgación necesarios a los fines sociales y humanos de la ciencia.

Bajo la denominación de "ciencias del ambiente" se reúne a las disciplinas tradicionales con injerencia en su estudio; por ejemplo, la Academia Argentina de Ciencias del Ambiente reúne a las ya mencionadas medicina, ingeniería, química, biología, meteorología, geografía, urbanismo y derecho, entre otras. Por otro lado, existen libros, revistas especializadas, carreras de grado y posgrado, que hacen mención a la "ciencia ambiental" como a un campo disciplinar definido. Es común, además, la nominación de "ciencias ambientales", no quedando siempre claro si bajo la misma se incluyen a las ciencias del ambiente o a nuevas ramas de la ciencia ambiental.

Finalmente, según se anticipó en el planteo de este trabajo, si bien se ha reconocido lo ambiental como un campo práctico problemático, la existencia de la ciencia 
ambiental ha sido puesta en duda desde algunas posiciones teóricas (Leff, 2000a). Es decir que en torno a la confusión generada respecto a considerar lo ecológico dentro de lo ambiental o, por el contrario, lo ambiental dentro de lo ecológico, se originan imprecisiones en el fundamento teórico de las disciplinas. En general, se alude a lo ambiental o a lo ecológico, sin explicitar el supuesto adoptado respecto de cuál concepto incluye a cuál. Por una parte, esto se explica por el desconocimiento de la otra posición teórica distinta a la adoptada, dadas las circunstancias de investigación, comunicación y divulgación del área disciplinar de la que se trate. Por otra, como ya se expresó anteriormente, dada la ausencia de un fundamento epistemológico concensuado sobre lo ambiental.

Las imprecisiones aludidas dentro de las disciplinas, a su vez, se trasuntan a diversos aspectos teóricos, metodológicos y prácticos de la investigación en diálogo con otras áreas disciplinares, surgiendo planteos sobre la multidisciplinariedad, interdisciplinariedad y transdisciplinariedad respecto a los estudios sobre el ambiente. En síntesis, a la naturaleza compleja del ambiente se suma la variedad de enfoques desde las distintas disciplinas, lo que se traduce en abordajes mayoritariamente fragmentarios de su estudio y compresión, no obstante el profuso y conocido impacto de su reconocimiento en los últimos años.

\section{LOS ESTUDIOS SOBRE EL AMBIENTE Y LAS GAUSAS DEL DETERIORO AMBIENTAL}

Las evidencias del deterioro ambiental, como consecuencia de acciones humanas con efecto negativo sobre la naturaleza y el propio ambiente humano, originaron planteos en los aspectos no sólo científicos y éticos de las diversas disciplinas, sino también, en los sociológicos y normativos derivados. El conocimiento científico de las causas de ciertos deterioros conlleva la necesidad de la resolución de los problemas asociados, los que involucran acciones individuales y desde las instituciones, las que a su vez dependen de la correcta divulgación de los resultados de las investigaciones, y la capacitación de los actores involucrados. Por ejemplo, en un estudio realizado en la provincia argentina de Santiago del Estero sobre la gestión ambiental llevada a cabo por algunos municipios, se identificó, como principal causa de ineficiencias detectadas en la gestión, la falta de capacitación en los gestores responsables, por encima de condicionamientos económicos, o incluso, de la voluntad política de realizar una correcta gestión (Giannuzzo, Villaverde \& Leiva, 2007).

Las acciones desacertadas, a su vez, originan nuevas consecuencias de deterioro, sobre las que, en algunos casos, se centra la investigación. Esto crea una especie de feedback positivo entre aplicaciones incorrectas derivadas de una inexacta compren- 
sión de los resultados de las investigaciones y los nuevos estudios orientados a resolver los problemas creados por el mismo desconocimiento que los originó. De este modo, se crean espacios vacíos entre los desarrollos teóricos y las aplicaciones, cuya progresión incide, a su vez, en inhibiciones o retardos en la nueva generación de conocimientos necesarios a resoluciones puntuales o generales. Se producen, de este modo, ineficiencias entre la generación de conocimientos necesarios a la resolución de problemas y la resolución efectiva de los mismos, siendo las mismas ineficiencias las que generan nuevos conflictos que suman a la confusión y no a la resolución teórica y práctica de los mismos.

Es oportuno señalar que las ineficiencias observadas entre el conocimiento y su aplicación dependem de dos factores esenciales: la eficiencia en la transferencia del conocimiento y el accionar ético en los diversos contextos y desde los diversos actores implicados.

Como ejemplo de lo antedicho, pueden mencionarse las metodologías de diagnóstico y gestión ambiental local, las que suelen ser importadas, adaptadas o desarrolladas desde algún área disciplinar. El desconocimiento de la realidad local, de indicadores de otras áreas disciplinares, tanto como la complicada aplicabilidad relacionada al diseño de la metodología en sí, a la capacitación de quienes deberían aplicarla, a la falta de recursos originan fallas de consecución práctica que confluyen en nuevos estudios de desarrollos metodológicos que no llegan a ser aplicados, o que son poco eficientes respecto al fin para el que fueron concebidos. Es común advertir, además, que muchas de tales metodologías no están centradas en la gestión ambiental de la unidad socio-ecológica, sino en un aspecto de la misma, el social o el ecológico, resultando en una utilidad parcial. Fallas en la transferencia del conocimiento de estas metodologías, unidas a debilidad política o ineficiencia para aplicarlas, son causa, a su vez, del feedback antes mencionado, debido a que las variables de estos componentes no son incorporadas por quienes las desarrollan, por desconocimiento o por limitaciones conceptuales y/o metodológicas.

Por su parte, la debilidad política e ineficiencia de aplicación en diversos ámbitos muchas veces se entrecruza con aspectos éticos personales o institucionales, como la deshonestidad y la corrupción. De este modo, advertimos hasta aquí que las soluciones dadas a los problemas que motivan las investigaciones dependen de una correcta difusión, transferencia y aplicación para incidir positivamente, desde un planteo de eficiencia temporal y de recursos, en la orientación de nuevas investigaciones y, fundamentalmente, para la resolución efectiva de los problemas. 


\section{INTENTANDO EL DISGERNIMIENTO SOBRE LA GONFUSIÓN:} LAS DISTINTAS AGEPGIONES DE AMBIENTE

Evidentemente, en un sentido contextual, lo "ambiental" y lo "ecológico" se han expandido como concepto, preocupación y acción, desde lo ético hasta lo normativo, en los diversos ámbitos de la vida humana como los institucionales, empresariales, industriales, y de la vida social e individual.

Sin embargo, como se expuso anteriormente, no siempre queda claro en los distintos ámbitos en los que se usa, incluyendo el científico, el filosófico, el de divulgación científica y el educativo, a qué se hace referencia con cada término. Usualmente lo ambiental es referido a lo humano y lo ecológico a lo natural, o ambos como a lo mismo. Es decir, se usan indistintamente ambiental y ecológico para referirse en forma conjunta a lo humano y lo natural, o, respectivamente, englobando lo ecológico dentro de lo ambiental, y viceversa.

$\mathrm{Al}$ respecto, y realizando un trabajo de análisis y síntesis, pueden identificarse las siguientes acepciones del término:

- aludiendo a un mismo concepto; por ejemplo, se habla de la preservación "ecológica" o "ambiental" en forma indistinta.

- aludiendo a conceptos distintos; por ejemplo, en ámbitos científicos, se suele hacer referencia a los efectos "ecológicos y ambientales" de los cambios climáticos. También, se hace alusión a "la ecología y las ciencias ambientales”, en especial en libros de texto.

- englobando lo ecológico dentro de lo ambiental; por ejemplo, en general, la bibliografía reseña como los tres pilares del desarrollo "ambiental" sustentable el "ecológico", el económico y el social. Algunos libros se titulan como "ciencia ambiental: ecología y medioambiente", o "ciencia ambiental: ecología y desarrollo sustentable".

- englobando lo ambiental dentro de lo ecológico; por ejemplo, se menciona a la ecología como el nexo entre las ciencias naturales y las sociales (cf. Odum, 1975).

Es común observar, además, que estas distintas acepciones de ambiente se usen indistintamente. 


\section{LAS AGEPGIONES DE AMBIENTE Y LAS DISGIPLINAS}

Según se expuso, pueden identificarse cuatro nociones con las que se suele hacer referencia a lo ambiental. Se intentará, ahora, fortalecer la idea de que tales nociones o acepciones, como hasta aquí las referimos, no son sino aspectos de un mismo concepto troncal. Esto puede explicarse, pues no se puede separar lo inseparable, sin que deje de ser según el concepto que lo une, y por el que son definidas las partes y el todo. Esto la tradición lo expresa como "el todo es más que la suma de las partes".

Sin embargo, según lo que se expone desde el inicio, no siempre la acepción a la que se hace mención con el uso del término queda claro, dándose generalmente por sobreentendida y, por esto, pudiendo originar confusiones en contextos en los que se lo conoce por una acepción distinta a la que es aludido el término, motivo por el cual resulta útil repasar brevemente su referencia en algunas disciplinas.

En la química y la ingeniería, por ejemplo, es común el uso del vocablo aludiendo a los componentes abióticos de los ecosistemas, agua, suelo, aire. En las ciencias sociales y humanas, el término suele utilizarse para indicar ciertas condiciones externas a un fenómeno bajo estudio; se hace referencia al ambiente económico, cultural, político. En biología y ecología, su uso alude a las condiciones bióticas y abióticas en las que vive un organismo, población o comunidad, es decir, al conjunto de influencias del exterior sobre los mismos. En ecología humana, es considerado como el conjunto de parámetros externos que, en forma directa o indirecta y a corto plazo, pueden tener influencia en la calidad de vida del hombre.

Desde el derecho, el ambiente ha sido descrito, por ejemplo, como el producto de la interrelación de los subsistemas naturales, económicos y sociales (cf. Sisto, 1999). En este sentido, el concepto suele ser acotado como ambiente social, sociocultural, natural, haciendo referencia a los subsistemas en interrelación o, por el contrario, reconociéndolos como entidades autónomas identificables y contrastables.

En áreas como la economía y la economía ecológica, el ambiente es referido como entorno o medio, como subsistema dentro de la interrelación de los sistemas naturales y sociales o, con menor frecuencia, en acuerdo a la acepción que aquí procura enfatizarse, el ambiente como la integridad plasmada por los subsistemas naturales y culturales. Otra posibilidad, muy extendida en las áreas mencionadas y otras, es la homologación del ambiente con la naturaleza.

Es oportuno recordar que el concepto de ambiente como aquí se enfatiza incorpora y admite indisociablemente la tecnosfera, es decir, el mundo de las invenciones y de la cultura humana (cf. Enkerlin, 1997), el mismo hombre como ser vivo y ambos a la vez en interacción con la naturaleza. 
Evidentemente, la confusión advertida en torno a lo ecológico y lo ambiental está asociada a la transformación histórica del concepto de ambiente. Es conocido que la acepción más ampliamente consensuada en la actualidad evoluciona desde concebir a los elementos físicos, químicos y biológicos, que rodean a los seres vivos espacialmente, hasta involucrar las condiciones socioculturales y la sucesión del tiempo, que comprometen el entorno de los seres vivos y las actividades humanas, considerando central los efectos de sus interrelaciones sobre la vida.

Desde la educación ambiental, se ha aludido a que el uso didáctico de la naturaleza está relacionado con el desarrollo histórico del concepto de ambiente y el enriquecimiento semántico que origina el uso del término "medioambiente". Desde una visión pasiva de la naturaleza, como medio para satisfacer las necesidades del hombre, ésta pasa a ser ambiente, lo que lo rodea y, a la vez, lo que condiciona incluso su supervivencia (cf. Villaverde, 1988; Meinardi \& Chion, 1999). Sin embargo, expresado así, el papel central de la tecnosfera o de las actividades humanas queda simplificado. Además, el hecho de que el uso del término constituya un enriquecimiento semántico es puesto en duda por el hecho de que algunos textos de ecología lo refieran con anterioridad, en el sentido "pasivo".

Otro aspecto interesante, que ayuda a dilucidar lo hasta aquí expuesto, lo muestra oportunamente un ensayo sobre epistemología de la biología que se refiere a la relación entre los seres vivos y su medioambiente, enfatizando que no hay organismos sin medioambiente, que éstos no lo experimentan sino que lo crean, lo construyen a partir de partes y piezas del mundo físico y biológico y mediante su actividad propia. Es decir que no hay un medioambiente en algún sentido independiente y abstracto (cf. Lewontin, 1984; Martínez, 1997).

La cita muestra que la acepción primera del concepto sigue siendo la misma, lo que varía es el alcance que le da al término, la relevancia que adquieren las actividades del hombre, como organismo natural y como hombre en el sentido cultural, en la modificación del "ambiente". En este punto, es importante remarcar que lo humano es comprendido en este trabajo como parte de lo natural, pero distinguido por la singularidad que expresa la emergencia de lo cultural.

Desde la nueva corriente denominada epistemología ambiental, queda claro que el ambiente es el campo de relaciones entre la naturaleza y la cultura (cf. Leff, 2004), es decir, que lo que se redefine es el vínculo dinámico que decide entre ambas las mutuas afectaciones observables.

Ahora bien, partiendo de una concepción de ambiente como una construcción conceptual referida a la interacción humanidad/naturaleza como un conjunto indisoluble, dadas las mutuas interacciones de sus componentes, se percibe que lo ambiental comprende lo natural, lo social y lo humano. Y el estudio del hombre como "ser 
humano" comienza a ser campo de estudio de las ciencias humanas y la filosofía, mostrando el aporte de las humanidades (cf. Tuana, 2007). Es decir, lo ambiental, como ya se lo expresó, es objeto plausible de estudio de todas las disciplinas del conocimiento, no sólo de la ecología, por lo que en este trabajo se prefiere la noción en la que lo ambiental engloba lo ecológico. Si bien, sin dudas, la ecología se erige como ciencia madre de los estudios sobre el ambiente.

Según se expuso anteriormente, en los dominios mencionados aparecen planteos que se presentan como relevantes en el momento de decidir un adecuado fundamento de las disciplinas implicadas. Ello configura una problemática de amplia índole que abarca tanto aspectos teóricos como prácticos en la enseñanza, investigación, tanto de grado como de posgrado, y en las aplicaciones de los resultados obtenidos. Eso define la necesidad de esclarecimiento conceptual al respecto. Paralelamente, la discusión acerca de distintos aspectos de la interdisciplinariedad y la transdisciplinariedad respecto a los estudios sobre el ambiente ocupa a las diversas disciplinas involucradas, existiendo abundantes planteos y propuestas (cf. Crowards, 1997; Goody, 1996; Lynch \& Wells, 1998; Lockwood, 1999; Pagano, 2010; Pawson \& Dovers, 2003; Spash, 1999). Por ejemplo, algunos investigadores relacionan interdisciplinariedad y dialéctica en la construcción de un conocimiento ambiental (Narváez, 2002); otros elaboran nuevas propuestas de herramientas conceptuales para la construcción de las ciencias ambientales (Rohde, 2010). Sin embargo, el esclarecimiento aún tarda en alcanzarse, y las resistencias en el diálogo interdisciplinar dificultan la fluidez en las influencias recíprocas.

Eso se comprende, como se lo intentó mostrar, por un lado, por lo complejo del objeto de estudio en sí, el ambiente y, por otro, por la diversidad de conceptos y métodos en las distintas disciplinas con injerencia en su estudio. La literatura refleja múltiples variantes relacionadas con perspectivas históricas, epistemológicas, metodológicas y valorativas de abordaje desde cada una (cf. Carter, 200o; Faber, Manstetten \& Proops, 1992; Goody, 1996; Mueller, 2001; Munda, 1997; Spash, 1999; Woodgate \& Redclift, 1998). Intentando un abordaje práctico, se pueden observar distintos tipos de estudios:

- estudios del ambiente desde las distintas disciplinas, cada una procurando el arribo a uno o varios aspectos desde la perspectiva de su área de conocimiento. Por ejemplo, el estudio de la biología del efecto de ciertos contaminantes sobre los organismos; el estudio de la química sobre el tratamiento de contaminantes; el estudio de la ingeniería sobre la optimización de los diseños de plantas de tratamientos; el estudio de la geografía sobre la localización de fuentes contaminantes y su impacto en el paisaje; el estudio de 
la economía sobre ciertos procesos productivos asociados a la producción de materiales contaminantes; el estudio de la política para instrumentar estrategias de gestión de residuos y el estudio desde la educación en procura de cambios de hábitos en la población para minimizar su producción. En este tipo de estudios, si bien los objetivos responden a algún o algunos aspectos de la problemática ambiental, se llevan a cabo sin implicar innovaciones significativas respecto a la estructura tradicional de cada disciplina. - estudios interdisciplinarios en los que, por ejemplo, un grupo de representantes de distintas disciplinas trabaja integradamente para resolver la problemática de la contaminación ambiental en una región determinada. En estos estudios cada disciplina sigue trabajando según su estructura fundamental, pero en diálogo con otras disciplinas con las que se procura además un consenso respecto de los objetivos y aspectos conceptuales y metodológicos que favorezcan la interrelación. En la actualidad, los aspectos interdisciplinarios son motivo de preocupación de las disciplinas que estudian el ambiente (cf. Goody, 1996; Lockwood, 1999; Narváez, 2002; Pagano, 2010; Pawson \& Dovers, 2003; Flores \& Zepeda, 2010).

- estudios ambientales que integran las áreas específicas de la temática ambiental de las distintas disciplinas en núcleos conceptuales que, a su vez, representan nuevas áreas. Por ejemplo, la contaminación ambiental sería un núcleo conceptual que, a la par de otros núcleos conceptuales como el cambio climático global, pertenece al dominio de la "ciencia ambiental". Esta ciencia, por ser joven, es dominio reciente de graduados de grado y postgrado en ejercicio, de los que se espera una fácil inserción en grupos interdisciplinarios por su formación plural respecto a las distintas disciplinas, y específica, respecto a la problemática ambiental.

- estudios ambientales que profundizan las relaciones entre los aspectos globales y los aspectos particulares de los núcleos conceptuales de la ciencia ambiental constituyendo, a su vez, especializaciones; esos estudios pertenecerían al dominio de las "ciencias ambientales". Un área disciplinar especializada sería, por ejemplo, la gestión ambiental. Otras especializaciones como la química ambiental o la ingeniería ambiental podrían concebirse como especializaciones de las disciplinas química e ingeniería, como especializaciones de la ciencia ambiental o como especializaciones producidas por el solapamiento de áreas de las disciplinas respectivas y la ciencia ambiental. A priori, en este trabajo, se las considera especializaciones de las disciplinas, por que estimamos que las especializaciones de la ciencia ambiental apenas comienzan a definirse. 


\section{El GongePto DE AMBiente considerado en la Conferencia de Estocolmo}

Como se expresó en un apartado anterior, la Conferencia de Estocolmo, en 1972, marca un hito en la divulgación universal del concepto del "medioambiente humano". $\mathrm{Al}$ considerarla por partes, esa definición comprende el conjunto de elementos físicos, químicos y biológicos, es decir, el conjunto de condiciones bióticas y abióticas al que alude, particularmente, la ecología en su primera acepción de ambiente, articulando además los factores sociales.

La expresión "factores sociales" incluye en forma concisa las variables sociales, culturales, económicas, políticas, tecnológicas y otras, entramadas en la dimensión humana, pero no siempre observadas como paralelas en sus diversas poblaciones. Es conocido que, al comparar poblaciones humanas, pueden identificarse semejanzas entre aspectos sociales, culturales, económicos, en forma conjunta o aislada, sin que esto implique necesariamente que se verifiquen semejanzas afines en los demás. Dos poblaciones pueden caracterizarse por poseer rasgos culturales comunes, pero utilizar distinta tecnología, y viceversa, dos poblaciones caracterizadas por rasgos culturales distintos pueden utilizar una misma tecnología.

El nexo establecido, "capaces de causar efectos directos o indirectos", trasciende la definición de ecosistemas, reseñada como el conjunto de interrelaciones entre los componentes bióticos y abióticos, ya que sesga hacia los efectos sobre los seres vivos y las actividades humanas la doble dirección de la neutralidad de los términos, influencias e interrelaciones, de uso generalizado en ecología. A su vez, los efectos que interesan al concepto son sobre los seres vivos y las actividades humanas, es decir, sobre la vida en general, y sobre la calidad de vida humana, en particular, al considerar sus actividades.

Resulta interesante resaltar que, al hacer alusión a los seres vivos y las actividades humanas, se comprende al hombre dentro del conjunto de los seres vivos como uno más y, además, se discierne su presencia a través de sus actividades. Las actividades hacen referencia a su condición humana, es decir, que el hombre no sólo concierne como ser vivo sino también como ser humano. Con simpleza, esta expresión reconoce la condición singular humana, sin contraponerla o ensalzarla respecto a otras expresiones de vida, sino incluyéndola.

Las acotaciones, "directos o indirectos" y "a corto o largo plazo", denotan el reconocimiento de la complejidad ambiental. Por la forma equilibrada en la que está expresada y la circunstancia en la que fue presentada, en este trabajo se considera que esta significación es la que subyace en la construcción actual del perfil de la ciencia ambiental. Sin embargo, la acotación de "humano" admite la interpretación de que 
habría, también, un "medioambiente no humano", aún cuando la definición está referida a los seres vivos y las actividades humanas, es decir, a todos los seres vivos, incluyendo al hombre. La acotación de "humano", entonces, se comprende como involucrando junto a los factores físicos, químicos y biológicos a los factores sociales como factores del entorno. Es decir, al involucrar a la sociedad humana como generadora de cambios en el entorno de los seres vivos, incluye los ocasionados a la propia especie.

De este modo, se refuerza la idea de la existencia de un "medioambiente no humano", que se definiría en relación al conjunto de sólo los factores físicos, químicos y biológicos, y los seres vivos "no humanos". Es decir, sin la inclusión de los factores sociales, que caracterizan a la "especie" humana, y sin su presencia en tales sistemas. Este "medioambiente no humano" se identificaría con naturaleza "pura”, es decir, con ecosistemas naturales no intervenidos por el hombre en alguna región prístina del planeta. Pero, el actual ejemplo del cambio climático con sus efectos globales o el de la movilidad atmosférica de ciertos contaminantes orgánicos nos orientan a considerar que no hay ecosistemas que no hayan sido impactados, en mayor o menor medida, por el hombre.

Además, aunque resulte elemental, resulta oportuno señalar la conocida paradoja que crea la evolución cultural, contraponiendo al hombre frente a la naturaleza, siendo él mismo naturaleza. Es decir, procede de la naturaleza, se sustenta de ella, y sus restos retornan a ella como los de los demás seres vivos. La vida y la muerte de los hombres están inscriptas en el "código", entiéndase, misterio de la naturaleza. La historia de la ciencia, más allá de las distintas corrientes filosóficas y creencias, muestra que lo que la humanidad conoce es sólo una pequeña parte de lo que quisiera conocer. Sin contar que el conocimiento científico mismo no alcanza para resolver las cuestiones fundamentales que la humanidad querría resolver. En los últimos decenios, a esto se suma el grado de incertidumbre sobre problemas que la propia evolución cultural creó; y, a la vez, es capaz de advertir, respecto a su ambiente, calidad de vida, y supervivencia.

\section{LA INTEGRAGIÓN DE LAS DISGIPLINAS}

EN LOS ESTUDIOS SOBRE EL AMBIENTE

La substitución matricial de lo natural por lo ambiental, es decir, la substitución de los estudios sobre la naturaleza por los estudios de la interacción humanidad-naturaleza o, como se presentó al principio, el reconocimiento del ambiente como objeto complejo de estudio, fue percibida en las distintas disciplinas con la consecuentes necesidad y búsqueda de la interdisciplinariedad y transdisciplinariedad. Esta segunda etapa ha sido señalada como la emergencia de las ciencias ambientales en un nivel epistemo- 
lógico, más precisamente como una segunda revolución epistemológica (cf. Rhode, 2010), en alusión a los tradicionales conceptos elaborados por Kuhn (1962). Más recientemente, la mayoría de los filósofos coinciden en que luego de una revolución científica, los conceptos científicos cambian su significado.

Kuhn (2002), al referirse a La estructura de las revoluciones científicas, recuerda la distinción entre desarrollos que simplemente aumentan el conocimiento y desarrollos que exigen abandonar parte de lo que se había creído hasta entonces. Como resultado de nuevas reflexiones, el mencionado autor prefiere distinguir entre desarrollos que requieren un cambio taxonómico local y los que no lo requieren, y agrega que tal modificación permite una descripción de lo que sucede durante el cambio revolucionario, significativamente más matizada que la que había brindado anteriormente. Además, actualiza la idea de inconmensurabilidad, la que se convierte en una especie de intraducibilidad, localizada en una u otra área en la que dos taxonomías léxicas difieren.

Kuhn refiere que el paralelo biológico del cambio revolucionario no es la mutación, sino la especiación, considerando que, después de una revolución, existen más especialidades o campos de conocimiento. Los mencionados cambios de taxonomía locales o esquemas conceptuales originan especiaciones, ya sea porque una nueva rama se separa del tronco vital, o bien porque surge una nueva especialidad en un área de aparente solapamiento entre dos especialidades preexistentes. El compartir categorías taxonómicas, al menos en un área de discusión, es prerrequisito para una comunicación sin problemas. El autor añade que esta segunda clase de escisión que suele ser celebrada como una reunificación de la ciencia, muchas veces se convierte en más una especialidad separada, y dado que la especiación impide la comunicación fluida entre especialidades, ésta no resulta una condición benigna para quienes valoran la unidad del conocimiento. Sin embargo, considera que la especialización y la reducción del campo de competencia son un medio eficaz para obtener herramientas cognitivas cada vez más potentes. Esto vuelve a ser enfatizado al expresar que muy probablemente sea precisamente la especialización, consecuencia de la diversidad léxica, lo que permita que las ciencias, consideradas colectivamente, solucionen los rompecabezas planteados por un ámbito de fenómenos naturales más amplios que el que una ciencia léxicamente homogénea podría abarcar.

De acuerdo a lo expuesto, puede aventurarse una analogía. Desde una concepción de la complejidad, podrían pensarse los desarrollos como una alteración de un fragmento organizacional del mundo cognitivo que se propaga a través de los elementos, las interrelaciones entre los elementos, el conjunto de elementos, propendiendo, a su vez, a reorganizaciones. De acuerdo al momento histórico, a la extensión y forma de la alteración, a la propiedad de resiliencia, a la propia dinámica de evolución cognitiva, se darán posibles grados de cambio en la estructuración, pudiendo ocurrir, even- 
tualmente, cambios taxonómicos locales. Lo que suele ser difícil, como ocurre en la biología, es comprender o explicar el acoplamiento entre las macro y micro dimensiones de los mecanismos que producen los cambios taxonómicos.

Teniendo en cuenta las consideraciones de Kuhn, podemos expresar que el desarrollo del concepto ambiente implica cambios locales de taxonomía o de esquemas conceptuales, los que originan especiaciones, por ejemplo, las ya mencionadas, química ambiental e ingeniería ambiental. Pero la especiación no es promovida sólo en consonancia con el esquema conceptual en que lo ambiental incluye lo ecológico, sino con otro esquema posible en el que lo ecológico incluye lo ambiental. Así, coexisten la economía ambiental y la economía ecológica, la ética ambiental y la ética ecológica, la historia ambiental y la historia natural.

Este aspecto no es negativo, si se considera que la diversidad de estructuras conceptuales, al originar controversias, conduce a innovaciones en este orden a través de procesos de interpretación y aprendizaje (cf. Ransanz, 1997). Desde estas perspectivas los aspectos antes señalados de interdisciplinariedad y transdiciplinariedad no se resolverán a través de diseños metodológicos, sin antes lograr la comunicación entre las disciplinas que estudian el ambiente, es decir, dilucidando esquemas conceptuales compartidos.

Retomando la concepción de revolución científica, y en consonancia con la diversidad de argumentaciones posteriores a las presentadas por el aludido autor, puede redefinírsela como un cambio fundamental del modo en que se piensa la realidad (cf. Brown, 1984). Aquí se expresa que, en este sentido amplio, se coincide con quienes interpretan que el cambio de la matriz ecológica por la ambiental constituye una revolución científica, aunque no necesariamente ella satisfaga la estructura kunheana ortodoxa de ciencia normal-crisis-revolución-ciencia normal.

No obstante, teniendo en cuenta la disyuntiva respecto a que si la percepción nos proporciona hechos puros o si la percepción está determinada en gran medida por el conocimiento, las creencias y las teorías que ya se sustentan, entendemos que puede suceder que un perceptor puede ver, en diferentes ocasiones, un objeto de distintas formas y la forma del objeto puede variar desde dos observadores diferentes (cf. Brown, 1984). Entendemos que el objeto de estudio, el ambiente, puede responder a acepciones diferentes de acuerdo a la diferente información que sobre él tiene cada uno de los observadores, las distintas disciplinas. Sin embargo, el problema no radica en este aspecto cuando está clara la acepción a la que se hace referencia. El problema se expresa cuando es necesario confrontarlas dentro de un contexto de mayor alcance, como la interdisciplinariedad, en ámbitos de investigación, docencia o aplicación.

En este sentido, la idea no es imponer una acepción sobre otra, sino acordar su uso, a los fines de optimizar los estudios pertinentes. Dentro de la ecología, usar el 
término ambiente con el sentido con que es usado tradicionalmente en esta disciplina es correcto, en cambio, referirse al desarrollo ambiental sustentable considerando lo ambiental como lo natural es, obviamente, incorrecto.

El reconocimiento de la no traducibilidad completa entre esquemas conceptuales distintos es considerado indicio de pluralidad. En este sentido, se ha rescatado que la inconmensurabilidad no implica incomprensión y que la misma cumpliría un papel epistémico importante, promoviendo el progreso en el conocimiento (cf. Ransanz, 1997). De este modo, la aceptación de esta pluralidad de esquemas o estructuras conceptuales es una condición necesaria para el logro de diálogo entre disciplinas, que favorezca el alcance de las construcciones conceptuales necesarias para el trabajo integrado y el consecuente enriquecimiento de las áreas implicadas.

Desde las discusiones sobre la ciencia y la tecnología para el desarrollo sustentable, se expresa que la integración presenta planteos respecto a la conciliación de las diferentes disciplinas en el mismo marco, y a la conciliación en términos de la comprensión mutua entre perspectivas irreducibles. Este último punto es sostenido por algunos autores que consideran el desarrollo de una misma plataforma de creencias (al menos parcial) como una condición necesaria para el diálogo, pero no suficiente por sí mismo, mientras que otros insisten en que esto no puede ser automáticamente asumido o impuesto (cf. Modvar \& Gallopín, 2010).

Es oportuno remarcar que, como es sabido, la integración en los estudios ambientales no sólo presenta condicionamientos en el diálogo entre las disciplinas en la teoría, y entre los profesionales en la práctica, sino que también está referida al diálogo entre los distintos actores sociales involucrados. En estas situaciones, no sólo se trata de articular diferentes visiones del mundo pero también diferentes y legítimos objetivos. La reducción de la pluralidad de puntos de vista e intereses a un único formato, por ejemplo, a un modelo matemático, a una descripción o un único objetivo, no es ni posible ni deseable. El análisis de las condiciones, tanto objetivas como subjetivas, y la experimentación de los distintos enfoques, es un componente importante de la nueva especie de investigación a largo plazo que se define para la ciencia y la tecnología dirigida al desarrollo sustentable (cf. Modvar \& Gallopín, 2010).

Dado el planteo actual del desafío investigación-acción, y en un plano más cercano a la práctica investigativa, en este trabajo se consideran las problemáticas ambientales (deforestación, desertificación, contaminación ambiental, cambio climático, pérdida de la biodiversidad, depleción de la capa de ozono, problemáticas ambientales urbanas) como nexos conceptuales para los estudios sobre el ambiente. 


\section{RAGIONALIDAD Y GONSIDERAGIONES ÉTICAS}

Como ya se expresó, se ha entendido a la ecología como un vínculo entre las ciencias naturales y las sociales (Odum, 1975). Leff (2000b), particularmente, procura acercarse a la interrelación entre las ciencias biológicas y las sociales a través de la ecología. Ese mismo autor (Leff \& Brañes, 1994; Leff, 2004) propone como un instrumento integrador de las diversas disciplinas la racionalidad ambiental, en tanto opuesta a la racionalidad productiva o capitalista. Este concepto surge a partir del análisis de los efectos de la problemática ambiental sobre las transformaciones metodológicas y la movilidad de términos entre las diversas disciplinas involucradas.

Por su parte, desde la ecología, se propone el concepto de racionalidad ecológica, fundamentado en la estabilidad multidimensional de la biosfera. La estabilidad de la biosfera funcionaría como substrato a todas las otras racionalidades y sería el fundamento del equilibrio, incluso el económico (cf. Schor \& Demajorovic, 2010). Sin embargo, se alega, haciendo referencia al concepto de sustentabilidad, que ésta no será el resultado de internalizar una racionalidad ecológica dentro de los engranajes de los ciclos económicos (cf. Leff, 2000c).

En esas propuestas, lo ambiental y lo ecológico se presentan diferenciados, conduciendo a diferencias en las metodologías derivadas y a distintas consecuencias de aplicación en los diversos contextos. En relación a lo expuesto, y realizando un rápido repaso histórico, se puede expresar en un planteo general a priori que las distintas acepciones de "ambiente" se entraman con diversas concepciones de racionalidad, desde una racionalidad científica e instrumental, pasando por una racionalidad económica, hasta una racionalidad social. Éstas reflejan, a su vez, distintas percepciones de relación de la humanidad con la naturaleza.

Esa esencia plural de la esfera ambiental, si bien implica respeto por distintas posiciones y criterios, no tiene que entrañar necesariamente una posición relativista en el sentido de que se consideren igualmente válidos los distintos estándares de racionalidad aun considerando un marco teórico fundamentado (cf. Ransanz, 1997). El presente y el futuro de la humanidad y la naturaleza merecen el esfuerzo de un diálogo que logre, más allá de la forma de percibir su interrelación desde las distintas disciplinas y culturas, acuerdos cognitivos y éticos.

Según esto último, y aludiendo a la disyuntiva sobre la que suele debatirse respecto a la prioridad de la especie humana o de las demás especies sobre la vida, agregamos que podría juzgarse natural priorizar la defensa de individuos de nuestra misma especie, y humano considerarnos responsables de la protección de individuos de especies diferentes. 
La tecnosfera ha posibilitado a la especie humana ampliar su nicho a todo el planeta y ser capaz de competir con éxito respecto a cualquier otra especie; lo que aún no está claro es si le posibilitará también adaptarse a los cambios que ha propulsado, no sólo como ser vivo, sino también como ser humano y en armonía con el resto de los seres vivientes. Así, lo ambiental trasciende lo ecológico porque no sólo involucra al hombre como una especie más y a su singularidad expresada a través de sus actividades, sino como responsable de los cambios "ecológicos" que induce. Y tal responsabilidad atañe al dominio de su ser conciente. De otro modo, se ha aludido a que lo ambiental excede lo ecológico, haciendo referencia al componente normativo que involucra lo ambiental, reflejado en su relación con la calidad de vida de los seres humanos y el concepto de calidad ambiental (cf. Gallopín, 2000). En concordancia con lo antedicho, se plantea que una visión común acerca del futuro puede ser el objetivo que posibilite el diálogo (cf. Modvar \& Gallopín, 2010).

Las problemáticas ambientales han enfrentado a la especie humana a la asunción universal de la responsabilidad por el deterioro ambiental y, por ende, de su conservación, resultando en una nueva idea de hombre responsable respecto a sus relaciones con la naturaleza (cf. Giannuzzo, 2005).

\section{Sobre LA EXISTENGIA DE LA GIENGIA AMBIENTAL \\ Y SU status EPISTÉMICO}

Como se expresó, el estudio de sistemas ambientales es el estudio de sistemas complejos. Están constituidos por elementos heterogéneos en interacción, lo que significa que sus subsistemas pertenecen a dominios materiales de diversas disciplinas (cf. García, 1994). Esta condición plantea los aspectos señalados sobre especializaciones, interdisciplinariedad y pluralidad. Es necesario consignar, además, que si bien se ha reconocido lo ambiental como un campo práctico problemático, la existencia de la ciencia ambiental ha sido puesta en duda desde algunas posiciones teóricas (cf. Leff, 2000a).

Respecto a su acepción, en la última década, algunos autores han acotado elaboraciones basadas en diversas conceptualizaciones, como población, actividades humanas, regeneración de los recursos, contaminación, intercambio de materia y energía (cf. Chiras, 1991; Miller, 1992; Cunningham \& Cunningham, 2002; Wright \& Nebel, 2002; Keller \& Botkin, 2007). Estos enfoques coinciden en dar prioridad a la interacción humanidad-naturaleza, aunque no siempre reflejando claramente sus implicancias sobre el status epistémico de este sector de la investigación científica. Se oscila en caracterizarlo como disciplina, interdisciplina o metadisciplina. 
En concordancia con presentaciones anteriores, en este trabajo, se la propone como una ciencia generalista, interdisciplinaria, heterogénea y en construcción. Es decir, una ciencia en estructuración de su esquema conceptual, del gran marco taxonómico que pueda compartir con todas las disciplinas implicadas en su ámbito. Así, la ciencia ambiental es referida como el conjunto de conocimientos y metodologías, provenientes de múltiples disciplinas, integrados con el objeto de comprender, predecir y accionar sobre las interrelaciones de las poblaciones humanas en su devenir histórico, social, cultural y tecnológico con la naturaleza y su evolución dinámica intrínseca. Los ámbitos de la ciencia ambiental se congregan en torno a los valores de respeto por la naturaleza y de responsabilidad en el logro de una organización que garantice la equidad, la calidad de vida y la supervivencia humana (cf. Giannuzzo, Rodríguez \& Viana, 2004).

De este modo, los planteos referidos sobre la necesidad de un enfoque transdisciplinario de los problemas ambientales para su resolución seríam uno de los basamentos para la existencia de la ciencia ambiental. La existencia de profesionales conocedores de las problemáticas en sus variados aspectos naturales, sociales, técnicos y, también, humanísticos, facilitarían el diálogo entre: (a) las investigaciones básicas y orientadas, y las aplicadas; (b) los resultados de las investigaciones, las acciones políticas necesarias, las organizaciones e instituciones implicadas, y las poblaciones humanas y de demás seres vivos afectadas por las distintas situaciones; (c) los diversos profesionales especializados en los distintos aspectos de las problemáticas.

La ciencia ambiental, de este modo, sería una ciencia holista, por su marco referencial macro abarcador, integrador, multifacético, pero también, sintética, por articular las síntesis conceptuales y metodológicas de las distintas disciplinas que entienden, en los diversos aspectos, las problemáticas en sus aspectos micros o particulares. Se trata de una ciencia que basa sus objetivos de estudio en los efectos sobre los seres vivos como centro de las problemáticas ambientales. En consecuencia, es una ciencia que se perfila relacionando los conocimientos y metodologías inherentes: químicos, físicos, biológicos, toxicológicos, geográficos, climatológicos, ecosistémicos, propios de las llamadas ciencias exactas, físicas, naturales y de la tierra. Tales conocimientos son articulados, a su vez, a conocimientos y metodologías de las ciencias sociales y humanas, con el fin de gestionar tales problemáticas y en consecuencia de planificar, incluyendo las injerencias y derivaciones políticas, económicas y éticas, es decir, con el fin de prevenirlas, minimizarlas, remediarlas, normalizarlas. De este modo, el dominio de la ciencia ambiental es la intersección de las ciencias naturales, las sociales y las humanas, para el estudio, tratamiento, gestión y planificación de los problemas ambientales. 
Con respecto al ejemplo sobre el problema de contaminación abordado desde distintos objetivos de estudio, dentro de la ciencia ambiental, el núcleo conceptual de la contaminación ambiental está configurado sintéticamente por todos los aspectos de las ciencias naturales, sociales y humanas de su injerencia, pero su núcleo "práctico" está sesgado hacia las ciencias sociales y humanas sobre los efectos en los seres humanos y demás seres vivos y la gestión y planificación de tales efectos. Su dominio alterna entre situaciones particulares o locales, y universales o globales.

Creemos que una mayor precisión conceptual cimentada sobre un marco compartido por las disciplinas que estudian el ambiente, incluida la ciencia ambiental, y los distintos actores involucrados en las problemáticas ambientales favorecerá el refinamiento de las metodologías tendientes a disminuir la fragmentación de las investigaciones concernientes y las aplicaciones para su resolución.@

Agradecimientos. Al Prof. Víctor Rodríguez y a la Dra. Marta de Viana por sus aportes y sugerencias para la redacción de este trabajo.

\author{
Amelia Nancy Giannuzzo \\ Professora Adjunta, Facultad de Ciencias Forestales, \\ Universidad Nacional de Santiago del Estero, Argentina. \\ nancygia@unse.edu.ar
}

\begin{abstract}
The existence of an environmental science is recognized in books, journals of science as well as in undergraduate and graduate studies. Its existence, however, is unknown either literally or indirectly when, for instance, its contribution to topics connected to the science and technology of sustainability is not considered. This background is presented in this paper and connected to its objective, which is to elucidate the existence and structure of the environmental science. To this goal, I analyse the relationship of the disciplines with the complex dimension of the environment as their object and the methodological aspects derived from it. In order to contribute to such conceptual clarification, I identify the various meanings of environment usually included in the bibliography. In addition, I discuss other aspects connected to multidisciplinarity, interdisciplinarity and transdisciplinarity together with the epistemic status of the environmental science. I conclude that a higher conceptual accuracy grounded upon a framework shared by the disciplines studying the environment, including the environmental science, and the various actors involved in environmental questions will favor the refinement of the methodologies tending to diminish the fragmentation of related research and the applications for their resolution.
\end{abstract}

KEYWORDS • Environmental studies. Environmental science. Interdisciplinarity. 


\section{REFERENGIAS BIBLIOGRÁFICAS}

Brown, H. I. La nueva filosofía de la ciencia. Madrid: Tecnos, 1984.

CARTer, A. Humean nature. Environmental Values, 9, p. 3-38, 2000.

CRowards, T. Nonuse values and the environment: economic and ethical motivations. Environmental Values, 6, p.143-67, 1997 .

Cunningham, W. P. \& Cunningham, M. A. Principles of environmental science: inquiry and applications. New York: Mc Graw-Hill Higher Education, 2002.

Chiras, D. D. Environmental science: action for a sustainable future. 3. ed. Redwood City: Benjamín/ Cummings Pub. Co, 1991.

Enkerlin, E. C. Ciencia ambientaly desarrollo sostenible. México: Internacional Thomson Editores, 1997.

Faber, M.; Manstetten, R. \& Proops, J. L. R. Humankind and the environment: an anatomy of surprise and ignorance. Environmental Values, 1, p.217-41, 1992.

FLoRes, R. P. \& ZePEDA, F. A. Interdisciplinariedad y transdisciplinariedad en los modelos de enseñanza de la cuestión ambiental. Cinta de Moebio, 15, 2002. Disponible en: <http://www.moebio.uchile.cl/ 15/frameso2.htm>. Acceso en: 13 feb. 2010.

Gallopín, G. Ecología y ambiente. In: LefF, E. (Org.) Los problemas del conocimiento y la perspectiva ambiental del desarrollo. México: Siglo xxi, 2000. p. 88-141.

García, R. Interdisciplinariedad y sistemas complejos. In: LefF, E \& Brañes, R. (Org.). Ciencias socialesy formación ambiental. Barcelona: Gedisa, 1994. p. 85-125.

Giannuzzo, A. N. El camino de las ciencias ambientales a las naturales: hacia la definición de las ciencias ambientales. In: Giannuzzo, A. N. \& Ludueña, M. E. (Org.). Santiago del Estero, una mirada ambiental. Santiago del Estero: Universidad Nacional de Santiago del Estero, 2005. p. 61-81.

Giannuzzo A.; Rodríguez, V. \& Viana, M. Los conceptos de ecología y ambiente y la relación entre ecología y ciencia ambiental. Actas de la II Reunión Binacional de Ecología argentino chilena, Mendoza, Argentina, 2004.

Giannuzzo, A. N. \& Ludueña, M. E. (Org.). Santiago del Estero, una mirada ambiental. Santiago del Estero: Universidad Nacional de Santiago del Estero, 2005.

Giannuzzo, A. N.; Villavverde, A. \& Leiva, M. Evaluación de la problemática ambiental de ciudades en la provincia de Santiago del Estero. Segundo Simposio Internacional de Investigación. Jujuy: Argentina, 2007. p. $15^{3 .}$

Gomez, A. V. (Org.). Racionalidad y cambio científico. México: Editorial Paidós Mexicana, 1997.

Goody, J. Man and the natural world: reflections on history and anthropology. Environment and History, 2, p. 255-69, 1996.

Keller, E. A. \& Botkin, D. B. Essential environmental science. Hoboken: John Wiley \& Sons, 2007.

Kunn, T. S. The structure of scientific revolutions. Chicago: University of Chicago Press, 1962.

. El camino desde la estructura. Ensayos filosóficos 1970-1993, con una entrevista autobiográfica. Barcelona: Paidós, 2002.

LefF, E. \& Brañes, R. (Org.). Ciencias sociales y formación ambiental. Barcelona: Gedisa, 1994.

LefF, E. (Org.). Los problemas del conocimiento y la perspectiva ambiental del desarrollo. México: Siglo xxI, 2000.

Ambiente y articulación de ciencias. In: Los problemas del conocimiento y la perspectiva ambiental del desarrollo. 2. ed. rev. México: Siglo xxi, 2000a. p. 27-87.

Epistemología ambiental. São Paulo: Cortez, 200ob.

.Educación para el desarrollo sustentable en ciencia, cultura y sociedad. Buenos Aires: "Marina Vilte"/ CTERA, 2000c. 
LefF, E. Educación ambiental: perspectivas desde el conocimiento, la ciencia, la ética, la cultura, la sociedad y la sustentabilidad. Primer Congreso Nacional de Educación Ambiental para el Desarrollo Sustentable de la Argentina, 2004.

Complejidad, racionalidad ambiental y diálogo de saberes. Actas do I Congreso Internacional Interdisciplinar de Participación, Animación e Intervención Socioeducativa, p. 1-12, 2006. Disponible en: $<$ www.mma.es/portal/secciones/formacion_educacion/reflexiones/2006_oreleff.pdf $>$. Acceso en 11: feb. 2010.

Lewontin, R. La diversidad humana. Barcelona: Prensa Científica, 1984.

Lynch, T. \& WeLLs, D. Non-anthropocentrism? A killing objection. Environmental Values, 7, p.151-63, 1998.

Lockwood, M. Humans valuing nature: synthesizing insights from philosophy, psychology and economics. Environmental Values, 8, p. 381-4,01, 1999.

NARVÁEz, C. E. M. Hacia una interdisciplinariedad dialéctica en la construcción de un conocimiento ambiental. Simposio Internacional El Reto Ambiental, Responsabilidad Compartida: Universidad Veracruzana, Boca de Río Veracruz, 2002.

Martínez, S. De los efectos a las causas: sobre la historia de los patrones de la investigación científica. México: Paidós Mexicana, 1997 .

MAYer, M. Educación ambiental: de la acción a la investigación. Enseñanza de las Ciencias, 16, 1, p. 217-31, 1998.

Meinardi, E. \& Chion, A. R. Teoría y práctica de la educación ambiental. Buenos Aires: Aique Grupo Editor, 1999 .

MilLER, G. T. Living in the environment: an introduction to environment science. 7 . ed. Belmont: Wadsworth Publishing Copany, 1992.

Modvar, G. \& Gallopín, G. G. Sustainable development: epistemological challenges to science and technology. Chile: United Nations Publications, 2005. Disponible en: <http://www.eclac.cl/publicaciones/Medio Ambiente/3/LCL2273P/lcl2273.pdf >. Acceso en: 13 feb. 2010.

Mueller, C. C. Economics, entropy and the long term future: conceptual foundations and the perspective of the economics of survival. Environmental Values, v. 10, p.61-384, 2001.

Munda, G. Environmental economics, ecological economics, and the concept of sustainable development. Environmental Values, 6, p. 213-33, 1997 .

Odum, E. P. Ecology: the link between the natural and the social sciences. 2. ed. London: Holt Reinhart \&Winston, 1975 .

Pagano, P. La filosofia ambientale come interazione dialettica tra science umane o science naturali. Sistema Naturae, 5, p. 193-217, 2003. Disponible en: <www.biologiateorica.it/systemanaturae/art2003/ Pagano.pdf $>$. Acceso en: 13 feb. 2010.

Pawson, E. \& Dovers, S. Environmental history and the challenges of interdisciplinarity: an antipodean perspectiva. Environment and History, 9, p. $5^{3-75}, 2003$.

Ransanz, A. R. P. Cambio científico e incomensurabilidad. In: Gomez, A. V. (Org.). Racionalidady cambio científico. México: Paidós Mexicana, 1997, p.71-97.

Reвoratti, C. Una cuestión de escala: sociedad, ambiente, tiempo y territorio. Sociologías, 5, p. 8o-93, 2001.

Rонde, G. M. The revolution of the environmental sciences. 31st International Geological Congreso, Rio de Janeiro, Brasil, 2000. Disponible en: <http://www.philosophia-online.de/mafo/heft2001-04/ Rohde, \%2, Revolution.doc $>$. Acceso en: 13 feb. 2010.

Schor, T. \& Demajorovic, J. Interdisciplinaridade em educação ambiental: utopia prática, 2002. Disponible en: <http://www.anppas.org.br/gt/sociedade_do_conhecimento/Tatiana\%2oSchor.pdf $>$. Acceso en: 13 feb. 2010. 
Sisto, M. C. Z. El orden ambiental. Buenos Aires: Ugerman, 1999.

Spash, C. L. The development of environmental thinking in economics. Environmental Values, 8, p. 4.1335,1999 .

Tuana, N. Human-environment interactions: a plea for the humanities. Nature and Culture, 2, 2, p. 21022,2007

Villaverde, N. Educación ambiental. México: Anaya, 1988.

Woodgate, G. \& Redglift, M. From a 'sociology of nature' to environmental sociology: beyond social construction. Environmental Values, 7, p. 3-24, 1998.

Wright, R. T. \& NebeL, B. J. Environment science: toward a sustainable future. 8. ed. New Jersey: PrenticeHall, $2,002$. 